\title{
Determination of 4-nitrophenol in Water using Free-Standing Cu Nanowire Electrode
}

\author{
Xiaoping Zhang, Lingxiao Chen, Yifan Zheng, Haodong Tang, Zongjian Liu* \\ Institute of Industrial Catalysis, College of Chemical Engineering, Zhejiang University of Technology, \\ Hangzhou 300014, P.R. China \\ *E-mail: zjliu@zjut.edu.cn
}

doi: $10.20964 / 2018.06 .40$

Received: 26 January 2018 / Accepted: 20 March 2018 / Published: 10 May 2018

\begin{abstract}
While $\mathrm{Au}$ and $\mathrm{Ag}$ are widely used as the electrocatalysts for sensing 4-nitrophenol (4-NP), $\mathrm{Cu}$ is seldom studied probably due to the fact that its performance is not as good as those of Au and Ag. In this work, the electrocatalytic activity of a free-standing $\mathrm{Cu}$ nanowire $(\mathrm{CuNW})$ electrode for 4-NP reduction, as well as its performance as a sensor for detection of 4-NP in water, has been studied. The electrochemical impedance spectroscopy analysis indicates that, owing to its a porous network structure with CuNWs serving as both conductive substrate and electrocatalyst, the free-standing CuNW electrode possesses a much lower electron transfer resistance and a much higher electrochemical active surface area than a CuNW-modified glassy carbon electrode (GCE). A comparative study on the electroreduction of 4-NP at different $\mathrm{Cu}$ electrodes by cyclic voltammetry (CV) reveals that no reduction peak appears at a $\mathrm{Cu}$ wire electrode with low active surface area but fast charge transfer while a broad peak is observed at the CuNW-modified GCE with slow charge transfer but relatively higher active surface area. Benefitting from the combination of fast electron transfer and high active surface area, however, the 4-NP reduction peak at the free-standing CuNW electrode is narrow and positively shifted with its current being more than 30 times higher than that observed at the $\mathrm{CuNW}$-modified GCE. These features allow an investigation of this free-standing CuNW electrode as a 4-NP sensor via a simple electrochemical technique (namely CV), and the results obtained demonstrate that this sensor exhibits a linear range of 4 to $2200 \mu \mathrm{M}$, a sensitivity of $4.831 \mu \mathrm{A} / \mu \mathrm{M}$, and a detection limit of $1.0 \mu \mathrm{M}$ at a signal-to-noise ratio of 3 . Compared with the reported porous $\mathrm{Cu}$ modified graphite pencil electrode, our $\mathrm{Cu}$-based 4-NP sensor not only possesses a lower detection limit but also has a much broader linear range and higher sensitivity.
\end{abstract}

Keywords: Cu nanowires; Electrochemical sensor; Free-standing electrode; 4-Nitrophenol

\section{FULL TEXT}


(C) 2018 The Authors. Published by ESG (www.electrochemsci.org). This article is an open access article distributed under the terms and conditions of the Creative Commons Attribution license (http://creativecommons.org/licenses/by/4.0/). 